\title{
Research Square \\ How Inhaler Technique is Assessed in Children and Young People: A Scoping Review Protocol
}

Kathryn Claire Anne Ferris ( $\nabla$ kferris02@qub.ac.uk)

Queen's University Belfast https://orcid.org/0000-0001-5220-7466

\section{Paddy McCrossan}

Queen's University Belfast

\section{Michael D Shields}

Queen's University Belfast

James Paton

University of Glasgow

Dara O'Donoghue

Queen's University Belfast

\section{Protocol}

Keywords: Inhaler technique, Asthma, Paediatrics, Asthma control, assessment

Posted Date: December 20th, 2021

DOI: https://doi.org/10.21203/rs.3.rs-1135431/v1

License: (c) (i) This work is licensed under a Creative Commons Attribution 4.0 International License. Read Full License 


\section{Abstract}

\section{Background}

Asthma is the most common chronic childhood condition. Unfortunately, many children have poorly controlled asthma. Current guidelines strongly recommend that all asthma review appointments must include an assessment of the patient's inhaler technique. However, most guidelines do not provide information on how the healthcare professional should conduct this assessment. The aim of this scoping review is to explore the published literature on methods used to assess inhaler technique.

\section{Methods}

This scoping review will follow the frameworks founded by Arksey and O'Malley and the Joanna Briggs Institute guidelines. We will search MEDLINE, Embase, Cinahl and the Cochrane library for studies published from $1^{\text {st }}$ January 1956 to $30^{\text {th }}$ November 2021, on methods of assessing inhaler technique in children and young people aged 1 to 16 years of age with asthma. We will include randomised control trials, case control studies, cohort studies and retrospective studies which investigate methods used to assess inhaler technique in children and young people. We will include studies conducted in all areas where inhaler technique assessment occurs and studies conducted by all healthcare professionals who usually undertake inhaler assessments in practice.

Two reviewers will complete all screening and data extraction independently. Data will be extracted onto a charting table and a descriptive summary of the results presented.

\section{Discussion}

This scoping review will provide a broad overview of currently used methods to assess inhaler technique in children and young people with asthma. The analysis of which will allow us to consider how these methods might be used in clinical practice and research settings.

Scoping review registration

Open Science Framework (osf.io/e47sa).

\section{Background}

Asthma is the most common chronic childhood condition $(1,2)$. Unfortunately, many children have poorly controlled asthma (3). The Asthma UK Annual Asthma Survey 2020 showed that only $48.7 \%$ of children and young people aged 17 or under were receiving the basics of asthma care (4). These basics of asthma care included an annual review of their asthma with a trained healthcare professional (HCP), inhaler technique assessment and having an agreed personalised asthma action plan $(4,5)$. Patient education including empowering patients and their families to actively self-manage their asthma is fundamental to the basics of asthma care (2). 
It has been identified that poor inhaler technique and poor medication adherence are major contributors to poor asthma control. Ensuring that the basics of asthma care are in place should improve asthma control and reduce exacerbations (6).

Effective drug delivery of inhaled medications is reliant on correct inhaler technique (7). There are many different types of inhalers, each with its own instructions and critical required steps, currently prescribed by healthcare professionals (8). This makes it challenging for HCPs to both to ensure consistent initial training of correct inhaler technique and later to ensure consistent assessment of whether the patient is using the correct technique.

Current guidelines strongly recommend that all asthma review appointments should include an assessment of the patient's inhaler technique $(7,8)$. However, most guidelines do not provide information on how the healthcare professional should conduct this assessment. Two recent systematic reviews concluded that the assessment of inhaler technique within clinical studies was too heterogeneous, and this meant they were unable to perform a meta-analysis $(9,10)$.

The aim of this scoping review is to search the published literature on methods used to assess inhaler technique, to map the concepts used within these methods, exploring the advantages / disadvantages of each and to determine whether there are validated tools available to operationalise the assessments. We aim to explore the ways in which clinicians assess inhaler technique during clinical reviews and assessments.

These wide research objectives, especially mapping of previous literature, are best achieved through a scoping review. This work will be useful in helping to develop a standardised method of assessing inhaler technique to ensure accuracy and consistency of practice regardless of the inhaler device used.

\section{Methods}

This scoping review will be conducted in accordance with the scoping review methodological frameworks described by Arskey and O'Malley (11); Levac et al (12); Colquhoon et al (13); and the Joanna Briggs Institute reviewer's manual (14).

The protocol will be reported in accordance with the reporting guidance provided by the Preferred Reporting Items for Systematic Reviews and Meta-Analysis Protocol - Extension for Scoping Reviews (PRISMA-ScR) checklist (15) (additional file 1) and has been registered within the Open Science Framework platform (osf.io/e47sa).

The methodological frameworks for performing a scoping review will involve the following stages:

1. Define the research question.

2. Identify relevant published literature.

3. Selection of relevant literature. 
4. Extract and chart the data collected from the literature.

5. Collate, summarise and report the results.

6. Consultation exercise.

\section{Stage 1: Define the research question}

Inhaler technique assessment is a crucial part of all asthma review appointments $(7,8)$. We want to explore if there are standardised and validated methods of assessing inhaler technique to ensure accuracy and consistency of assessment.

Following a preliminary search of the literature and discussion amongst the research team (which includes experienced academics in the field of paediatric asthma), the following specific research question was decided upon:

In children and young people with wheeze and/or asthma, what methods are used to assess inhaler technique in clinical practice and are these methods valid and reliable?'

Allowing for the iterative nature of a scoping review, following the initial piloting process we will review and consider refining the research question including the terms within it.

\section{Stage 2: Identify relevant published literature.}

Under the guidance of the Queen's University Belfast (QUB) medical librarian the search will include a range of terms and keywords related to the research question.

The following search terms will be used:

Asthma OR wheeze OR viral induced wheeze.

AND

Child OR young people OR adolescent OR paediatrics OR pediatrics.

AND

Inhaler technique OR inhaler management OR inhaler method.

AND

PMDI OR MDI OR DPI OR Spacer

AND

Checklists OR score OR assess OR assessment OR criteria 
An initial pilot exercise will be undertaken using the online database MEDLINE before proceeding to a complete search of all available databases. The lead researcher (KF) will review 10 publications from this initial piloting exercise to ensure that we have chosen the most suitable search terms by considering the different concepts that each of these articles could be mapped to. For the MEDLINE search appropriate $\mathrm{MeSH}$ terms will be included.

The lead researcher may consider modifying the search strategy and/or research question at this stage once we have a greater knowledge of the literature. A draft search strategy for MEDLINE is included (additional file 2).

Once the pilot is complete and consideration of new search terms made, a full search of MEDLINE will be completed followed by a full search of the subsequent chosen online databases (Embase, Cinahl and the Cochrane library). These databases provide a comprehensive list of relevant literature across a range of disciplines. A search of the grey literature (using google scholar) and discussion with a group of paediatric asthma specialists will also be undertaken to identify any additional relevant literature. We will make a record of the total number of citations yielded by these search terms from each of the databases. Duplications will then be removed before screening.

\section{Stage 3: Selection of relevant literature.}

Eligibility criteria:

We will include studies published since the year 1956 when the pressurised metered-dose inhaler (pMDI) was first brought into clinical practice (16). We will include randomised control trials, case control studies, cohort studies and retrospective studies which investigate methods used to assess inhaler technique in children and young people.

Studies involving both adults and children together will be included as these will still be relevant to our research question if these methods have been employed in children and young people. We do not wish to be 'device specific' and so we will include studies of metered-dose inhalers and dry powder inhalers (DPI), with and without the use of spacer devices.

We will include studies which have taken place in the emergency department, the out-patient department, the hospital ward and/or the community including general practice as these are all areas where inhaler technique assessment occurs. We will include studies conducted by healthcare professionals who usually undertake inhaler assessments in practice, this will include trained doctors and those in training, trained nurses and those in training, pharmacists, and physician associates.

To include a breadth of knowledge in this area we will include conference abstracts, presentations and scholarly information that may not have been peer reviewed.

\section{Exclusion criteria:}


We will exclude studies which explore the use of nebulised therapies as this involves a different technique. We will exclude studies which involve adult participants only.

Publications not in English will be excluded due to time and resource constraints as we will not be able to accurately translate the data and translated articles can have an inaccurate interpretation from the original article.

\section{Screening}

Initial screening will be based on the title with further screening based on the abstract, to ensure they fulfil the eligibility criteria. This will be conducted by lead researcher KF. Documents not meeting eligibility criteria will be excluded from further analysis.

The remaining publications will undergo full text analysis by three reviewers, from the research team (KF, $\mathrm{PMCC}$ and DOD). These three reviewers will independently analyse the full text articles. In the case of uncertainty, the text will be re-evaluated by a fourth independent reviewer (JP or MDS). The final search results will be exported into Endnote ${ }^{\mathrm{TM}}$ at which point all duplicates will be identified and removed.

A flow diagram in keeping with PRISMA will be used to report the searches and inclusion/exclusion pathway.Data will be stored and charted using a Microsoft Excel spreadsheet.

\section{Stage 4: Extract and chart the data collected from the literature.}

Two reviewers (KF and PMcC) will complete all screening and data abstraction independently. Data will be extracted onto a data-charting table (see Table 1) to create a descriptive summary of the results.

The charting table will record the characteristics of the included studies and the key information relevant to the research question. Given the iterative nature of a scoping review this table will be subject to further development following a review of this process with the first 2 or 3 studies.

Authors will be contacted directly for data clarification as necessary.

Information to be included in the charting table:

- Study characteristics: Design, year of publication, journal, year study was conducted (or period of time), sample size, country, setting, profession(s) of those conducting the study and study aims.

- Participant characteristics: age (e.g., mean with standard deviation, range) and gender.

- Inhaler characteristics: device type, use of inhaler adjunct devices such as spacers.

- Outcome measures.

- Key findings.

- Methods used in the study to provide an assessment of inhaler technique. In what context these methods have been used and if there has there been an assessment of inter/intra observer 
variability.

- Whether the assessment method was validated or not. If it has been validated, how has this been done.

- Whether objective methods of assessing inhaler technique were used (e.g flow meter attached to inhaler). If yes- which.

- Study concept- as defined by research group following discussion.

At intervals during the data extraction and charting process, charted data will be compared and discussed to ensure consistency between reviewers and to enable iterative reflection on emerging themes and categories.

During the review process, any amendments to the protocol deemed necessary by the team will be recorded in the master protocol document with the reason for amendment noted.

\section{Stage 5: Collate, summarise and report the results.}

We will aim to create a visual map categorising inhaler type and the assessment tools used for each.

We will present the qualitative results as a 'map' of the data in a descriptive format and diagrammatic format by classifying them under main conceptual categories. Numerical data will be analysed using Microsoft excel and presented in table format.

\section{Stage 6: Consultation exercise}

The consultation exercise will be used to add value to and validate our findings. The consultation exercise will consist of a focus group with our research group PPI team and volunteers through Asthma UK Centre for applied Research (AUKCAR) advertisement; these key stakeholders will be children and young people and/or their parents and carers who will have experience of being taught how to use an inhaler.

The focus groups will be held on Microsoft teams. The authors will compile a selection of inhaler assessment tools for discussion during the consultation exercise and some pre-set questions will be used to initiate and guide the discussion. Discussions will be recorded and transcribed on Microsoft teams and transcriptions checked verbatim. Written consent will be obtained. Focus group findings will be presented and shared along with the scoping review findings.

\section{Discussion}

As inhaler technique assessment is a crucial part of all asthma review appointments $(7,8)$ we want to explore if there are standardised and validated methods of assessing inhaler technique to ensure accuracy and consistency of assessment. 
Inhaler manufacturers produce insert leaflets with inhaler technique guides based on the steps for optimised inhaler technique for their specific inhaler device. It is possible to enumerate each of the manufacturers guideline steps, expecting the patient to undertake all steps correctly. However, some steps are critical; where if this didn't happen there would be a critical drug delivery error and therefore, they are of more importance and weighting of steps should likely be considered.

This has led some to recommend a more global assessment based on impressions such as (17);

- good inhaler technique $=$ when the clinician thinks patient likely inhaled most of the medication

- partial = when patient has made some errors, but the impression is that some medication was inhaled.

- poor or incorrect $=$ when the patient makes critical errors, and the observer receives the impression that no or very little medication was inhaled.

However global assessment-based rating may be influenced by inter-observer and intra-observer variability so may not provide consistent and reproducible results.

This scoping review will be an important first step in providing a broad overview of currently used methods to assess inhaler technique in children and young people with asthma/wheeze. The analysis of which will allow us to consider how these methods might be used in clinical practice and research settings.

The authors acknowledge that this study has been limited to those publications in the English language; however, the broad nature of the research question should still allow us to capture a significant proportion of available literature.

The results of this scoping review will be disseminated in peer-reviewed publications and presentations at international and national conferences focusing on paediatric Asthma as well as with local healthcare professional teams leading in the care of children and young people with Asthma and other key stakeholders. This will also include dissemination through AUKCAR, our research team Personal and Public Involvement (PPI) partners will be involved in writing a research update and sharing results to patients and their families through the Asthma UK website and through their research update bulletin as well as through relevant social media forums (18).

\section{Declarations}

\section{Ethics approval and consent to participate}

Ethical approval has not been sought as this is a secondary study of already published and publicly available literature.

\section{Consent for publication}


Not applicable.

\section{Availability of data and material}

Not applicable.

\section{Competing interests}

None to declare.

\section{Funding:}

This scoping review forms part of an educational qualification (PhD) and is funded by Asthma UK as part of the Asthma UK Centre for Applied Research [AUK-AC-2012-01 and AUK-AC-2018-01] and Royal Belfast Hospital for Sick Children charitable funds.

\section{Author contributions:}

DOD, PMcC and MS conceived the idea for this scoping review. KF is completing a PhD at Queen's University Belfast, and this scoping review will form part of her PhD. KF wrote the first draft of this manuscript. DOD, PMcC, MS and JP reviewed the manuscript resulting in a revised final manuscript, which has been read and approved by all authors.

\section{Acknowledgements:}

We would like to thank Mr. Richard Fallis, medical librarian at Queen's University Belfast, for his time and guidance on establishing the online literature search.

\section{References}

1. Ferrante G, La Grutta S. The Burden of Pediatric Asthma. Frontiers in Pediatrics. 2018;6(186).

2. Royal College of Paediatrics and Child Health. State of Child Health. 2020 [Available from: https://stateofchildhealth.rcpch.ac.uk/evidence/long-term-conditions/asthma/\#ref-1.

3. Asthma UK. UK asthma death rates among worst in Europe | Asthma UK 2015 [Available from: https://www.asthma.org.uk/about/media/news/press-release-uk-asthma-death-rates-among-worstin-europe/.

4. Asthma UK. Annual asthma survey | Asthma UK: @asthmauk; 2020 [Available from: https://www.asthma.org.uk/support-us/campaigns/publications/survey/.

5. Levy ML. The national review of asthma deaths: what did we learn and what needs to change? Breathe. 2015;11(1):14-24.

6. de Groot EP, Kreggemeijer WJ, Brand PLP. Getting the basics right resolves most cases of uncontrolled and problematic asthma. Acta Paediatrica. 2015;104(9):916-21. 
7. (GINA) GifA. Global strategy for asthma management and prevention. 2021; 2021.

8. British Thoracic Society. BTS/SIGN158 British guideline on the management of asthma July 2019 [Available from: https://www.brit-thoracic.org.uk/quality-improvement/guidelines/asthma/.

9. Gillette C, Rockich-Winston N, Kuhn JA, Flesher S, Shepherd M. Inhaler Technique in Children With Asthma: A Systematic Review. Academic Pediatrics. 2016;16(7):605-15.

10. Normansell R. Interventions to improve adherence to inhaled steroids for asthma. Cochrane Database of Systematic Reviews. (4).

11. Arksey H, O'Malley L. Scoping Studies: Towards a Methodological Framework. International Journal of Social Research Methodology. 2005;8(1):19-32.

12. Levac D, Colquhoun H, O'Brien KK. Scoping studies: advancing the methodology. Implementation Science. 2010;5:69-77.

13. Colquhoun HL, Levac D, O'Brien KK, Straus S, Tricco AC, Perrier L, et al. Scoping reviews: time for clarity in definition, methods, and reporting. Journal of Clinical Epidemiology. 2014;67(12):1291-4.

14. Peters MD, Godfrey CM, Khalil H, McInerney P, Parker D, Soares CB. Guidance for conducting systematic scoping reviews. Int J Evid Based Healthc. 2015;13(3):141-6.

15. Tricco AC, Lillie E, Zarin W, O Brien KK, Colquhoun H, Levac D, et al. PRISMA Extension for Scoping Reviews (PRISMA-ScR): Checklist and Explanation. 2018:467.

16. Crompton G. A brief history of inhaled asthma therapy over the last fifty years. Primary Care Respiratory Journal. 2006;15(6):326-31.

17. Patterson EE, Brennan MP, Linskey KM, Webb DC, Shields MD, Patterson CC. A cluster randomised intervention trial of asthma clubs to improve quality of life in primary school children: the School Care and Asthma Management Project (SCAMP). Arch Dis Child. 2005;90(8):786-91.

18. Asthma UK | Homepage: @asthmauk; [Available from: https://www.asthma.org.uk/.

\section{Supplementary Files}

This is a list of supplementary files associated with this preprint. Click to download.

- Additionalfile1PRISMAScRFillableChecklistKFerris.docx

- Additionalfile2KFerrisDraftsearchforMEDLINE.docx 